\title{
Reflexiones Docentes sobre Inclusión Educativa. Relatos de Experiencia Pedagógica sobre la Diversidad Universitaria
}

\section{Teacher's Reflections on Inclusive Education. Stories of Pedagogical Experience about College Diversity}

\author{
Andrea Olmos Roa * \\ María Rebeca Romo Pinales \\ Laura María del Carmen Arias Vera \\ Universidad Nacional Autónoma de México
}

\begin{abstract}
En este documento presentamos desde una mirada crítica algunas reflexiones, problemas, incertidumbres y propuestas, surgidos en un proceso de documentación narrativa de experiencias pedagógicas, modalidad de indagación- acción- formación, a través de reflexiones críticas sobre relatos editados y compartidos entre docentes universitarios, realizado con el fin de analizar los sentidos construidos sobre la diversidad del estudiantado y los problemas de exclusión educativa. Abrimos con un análisis de algunas propuestas que se han dado al problema de la diversidad, como la integración escolar y social a los niños con algunas discapacidades, y las propuestas de educación inclusiva. Posteriormente discutimos algunas barreras que obstaculizan la formación de algunos estudiantes, particularmente ciertas creencias, representaciones y supuestos que como docentes hemos construido respecto a este proceso y que se concretan en las culturas, las políticas y nuestras prácticas escolares. Finalizamos con esbozos posibles, derivados del estudio, para integrar relaciones pedagógicas incluyentes, como el desnaturalizar y cuestionar algunos de los supuestos y representaciones que orientan muchas de las decisiones y prácticas cotidianas para retomar algunas apuestas de cambio para reconocer y valorar la otredad en nuestras universidades.
\end{abstract}

Descriptores: Inclusión educativa, Diversidad, Narrativa pedagógica, Otredad, Relatos de experiencia.

In this paper we present a critical view at some thoughts, problems, uncertainties and proposals arising in the process of narrative documentation of educational experiences, inquiry- action mode, done through critical reflections on stories edited and shared learning experience among university teachers, in order to analyze the senses built on the diversity of students and educational exclusion problems. We continue with a discussion of various proposals that have been given to the problem of diversity and social and school children with some disabilities integration, and proposals for inclusive education, as well as some barriers to the formation of some students, particularly certain beliefs, assumptions and representations as teachers have built on this process and which are specified in the cultures, policies and practices of our school. We finish with sketches possible, derived from the study, for an inclusive education, such as denature and question some of the assumptions and representations that guide many decisions daily practices and revisiting some betting educational transform to recognize and value the otherness in our universities.

Keywords: Educational inclusion, Diversity, Pedagogical narrative, Otherness, Experience story.

*Contacto: andreaolmos@gmail.com

ISSN: 0718-7378

www.rinace.net/rlei/
Recibido: 15/06/2015

$1^{\text {a }}$ Evaluación: 26/08/2015

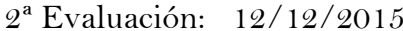

Aceptado: 20/01/2016 


\section{Introducción}

En la complejidad del mundo actual, la práctica docente en la universidad también se ha complicado demandando reflexiones y propuestas de cambio en la formación del profesor en múltiples dimensiones y escenarios, uno de los cuales implica responder al desafío de atender a la diversidad tanto estudiantil como de la cultura académica universitaria.

Históricamente en el nivel educativo elemental, para dar respuesta a esta problemática, las propuestas se centraron en los estudiantes con una tendencia clínica y asistencial, desde una mirada médica o biologicista que "patologizaba" las diferencias e identificaba a ciertos alumnos como "enfermos" y trataba de corregir su desviación a la norma. De esta manera se fue construyendo la imagen del sujeto "diferente-deficiente" (Skliar, 2005) dando como resultado la separación de los estudiantes en espacios educativos diferenciales. Posteriormente con el fin de evitar la segregación de ciertos alumnos surge el movimiento de integración a la escuela regular a niños con algún tipo de discapacidad, o de otras necesidades educativas esppeciales, con la intención de apoyarlos a través de una serie de recursos brindados por lo general por especialistas. Sin embargo, al establecer categorías diagnósticas para aquellos estudiantes segregados, se ha impedido cuestionar a la escuela y a la noción de alumnos promedio utilizado como referente de las prácticas educativas (Skliar, 2005). En este sentido, Castel (2004) nos alerta sobre "la trampa" que suponen muchas prácticas de integración escolar y social, cuando el trabajo se centra aparentemente, en ayudar a los excluidos, ya que lo que se logra es evadir políticas preventivas y sistémicas destinadas a impedir que la gente sea excluida.

Recientemente surge la propuesta de inclusión, que en algunos casos, al ser confundida con la integración y utilizarse como sinónimos (Ainscow, 2012) ha impedido considerar la totalidad de exclusiones y discriminaciones que se dan al interior de los sistemas educativos y que interfieren en el desarrollo de políticas integrales (Blanco, 2006). La autora sugiere que aunque en diversas escuelas se han producido procesos de cambio como consecuencia de la incorporación de alumnos con necesidades educativas especiales, el movimiento de integración no ha logrado impactar a los sistemas educativos de forma significativa. En general, se ha transferido el modelo de atención a la diversidad propio de la educación especial a las escuelas regulares, centrándose más en la atención individualizada de estos alumnos (programas individuales, estrategias y materiales diferenciados, etc.) que en modificar aquellos factores del contexto educativo y de la enseñanza que limitan la participación y el aprendizaje no sólo de los niños y jóvenes integrados, sino de todo el alumnado. A diferencia de la integración en algunos enfoques de la inclusión se considera que el problema no es el estudiante, sino el sistema educativo y sus instituciones. En especial ciertos factores que limitan el acceso, permanencia y el aprendizaje del alumnado como la escasez de recursos, la rigidez de la enseñanza, la falta de pertinencia de los currículos y la formación de los docentes, entre otros.

A pesar de que dicha propuesta de inclusión podría abarcar a todos los niveles educativos, los esfuerzos y políticas educativas con frecuencia se han centrado en el nivel elemental. En la universidad el panorama cambia en relación a la inclusión educativa, pues la política de acceso a la misma es un caso donde nuestros países no han dado respuesta. Por ello y dada su complejidad y diferentes aristas al problema es difícil abordarla en este espacio. En este caso, centraremos nuestra reflexiones a la problemática de atender e incluir a una diversidad de estudiantes que se matricularon, a 
raíz de los movimientos estudiantiles de hace décadas, a nuestras universidades diseñadas para una élite, las cuales han cambiado muy poco en esencia. Sabemos que ello implica un proceso lento y progresivo, en especial en la toma de conciencia y responsabilidad de los profesores y actores del proceso educativo, que requiere entre muchas otras cosas, eliminar aquellas prácticas docentes y de la gramática escolar que perciben la diversidad social, cultural, de capacidades e intereses con propuestas y análisis se centraron en los sujetos y no en las condiciones que impiden o favorecen la permanencia y éxito en la escuela.

Para comprender y analizar este reto incluyente que se presenta en las aulas universitarias es importante recordar que el uso de categorías, como alumno pobre, desertor, fracasado, indígena, en las teorías y discursos sociales hegemónicos, ha tenido una eficacia simbólica ya que como sujetos sociales llegamos a movernos sin dificultad con estas formas de nombramiento y percepción de lo social (Kaplan y García, 2006). Así, desde esta óptica, las diferencias en el estudiantado suponen puntos de partida a ser "compensados" a la hora de aprender. En las instituciones públicas, se hizo a través de becas o estrategias puntuales a grupos heterogéneos de alumnos indígenas, discapacitados o de escasos recursos, por ejemplo. Este proceso compensatorio ha dado lugar a un olvido a preocupaciones ligadas a los procesos de formación, a lo que cada estudiante aprende y, a la capacidad de todo el alumnado de aprovechar la oferta educativa. En este sentido, con la compensación se ha puesto en duda que la educación deba ser la misma para todos al introducir la idea de "adecuar" la educación a las condiciones sociales, psicológicas o biológicas que se ponen en juego en la formación y el aprendizaje.

El dilema, según Serra y Canciano (2006), es que si cancelamos la apertura de políticas hacia estos colectivos, argumentando que es una demanda de todos, sería una práctica injusta. Pero el problema contrario sería pensar que desarrollar prácticas inclusivas implica hacerlo sólo a determinados grupos, enfocando en ellos las propuestas de intervención, olvidando los procesos educativos que generaron su desventaja.

En este sentido encontramos los estudios relacionados con ciertas relaciones pedagógicas que pueden generar dicha desventaja como los realizados por Rosenthal (1994) acerca del efecto Pigmalión en la escuela que han mostrado la importancia de las expectativas, tanto positivas como negativas, de los docentes sobre sus alumnos, en especial con ciertos estudiantes sobre los cuales no se espera nada, lo cual refuerza su sensación inicial de que son poco competentes para las tareas escolares. Y de esta manera, como Kaplan y García (2006) argumentan los estudiantes marcados por procesos de exclusión de diverso tipo, da lugar a que tiendan a percibirse a sí mismos como causa última de su propio fracaso, valorándose en dependencia de cómo han sido reconocidos o desacreditados.

Afortunadamente se ha empezado a cuestionar la relación determinista entre las condiciones de enseñanza y ciertos contextos críticos, pues se ha demostrado que a pesar de que el escenario social sea difícil y hostil, la escuela o en particular, la universidad, puede ser un espacio donde se generen condiciones de apoyo y colaboración. Desde esta lógica, lo importante es eliminar esa mirada fatalista que se ha tenido por mucho tiempo, pues como se ha demostrado, las condiciones de enseñanza no están dadas o determinadas de manera lineal e inevitable desde el contexto social al que pertenecen los alumnos y, de esta manera podemos como docentes, pensar las posibilidades y 
alternativas que se abren si discutimos qué papel nos toca desarrollar como educadores en la construcción de dichas condiciones de formación y enseñanza. En este sentido, un aspecto que guía nuestro estudio es la convicción que como docentes podemos abrir espacios de reflexión y diálogo con los demás actores del hecho educativo, para cuestionar el discurso de la pedagogía tradicional sobre lo que se dice, pero sobretodo de lo que se calla, con el fin de poner en duda ciertas representaciones que contribuyen al establecimiento de medidas y procesos de estigmatización sobre ciertos alumnos. Para de ahí repensar y compartir nuestras prácticas educativas.

\section{La documentación de experiencias. Dispositivo metodológico}

Con el fin de analizar los sentidos construidos sobre la diversidad del estudiantado y los problemas de exclusión educativa, 25 profesores de las carreras de psicología y cirujano dentista de la Universidad Nacional Autónoma de México (FES Zaragoza), propusimos estudiar, revisar y reestructurar los supuestos culturales y psicológicos que definen nuestras prácticas, analizando cómo han influenciado nuestra forma de ser y de comportarnos en la práctica docente, para con base en ello proponer alternativas u opciones pedagógicas de acción, o cambio. Recuperamos procesos como la reflexión crítica (Saavedra, González y López, 2005), la formación horizontal (Suárez, 2006, y Suárez y Ochoa, 2005) y el aprendizaje transformativo (Mezirow, 1998) referido a esos cambios en nuestra perspectiva de significados, hábitos de pensamiento, marcos mentales, con el fin de volverlos más inclusivos, abiertos y reflexivos.

Este proceso de búsqueda y transformación lo construimos a través la documentación narrativa de experiencias pedagógicas, modalidad de indagación-acción-formación (Suárez, 2006), informada desde aportes teóricos y metodológicos de la investigación interpretativa en especial las versiones más críticas (Kincheloe, 2001), así como del método biográfico narrativo (Bolívar, Fernández y Domingo, 2001).

Así nos propusimos documentar, editar, intercambiar opiniones y compartir narraciones de experiencias pedagógicas, con el fin de fomentar y favorecer el debate y la reflexión sobre los procesos y retos pedagógicos en el aula. Así mismo, nos valimos de la propuesta de Freire (1997) para llevar a cabo una reflexión crítica en un proceso continuo de debate y reflexión sistemática sobre los relatos de experiencias editados y compartidos, que dieron lugar a múltiples interrogantes y temas sobre la vida en las aulas universitarias enmarcadas en ciertas condiciones políticas, sociales y económicas de nuestra universidad y sociedad mexicana. Esta experiencia fue útil para clarificar, resignificar y modificar nuestra práctica educativa a partir de la construcción de nuevos pensamientos emanados de la propia práctica (Duhalde, 2008), ya que al poner por escrito las vivencias mediante el relato, se recuperan de la memoria y se hacen visibles los pensamientos moldeados por la gramática cultural y es en la reflexión crítica donde puede ocurrir el cambio.

En particular, nos interesaba identificar y tratar de eliminar barreras o limitantes, principalmente aquellas vinculadas a ciertas creencias, actitudes, posturas, formas de conocer, de pensar y de actuar que como docentes hemos construido respecto a este proceso y que se pueden concretar en las culturas, las políticas y las prácticas escolares con los estudiantes universitarios. 
Iniciamos el análisis sobre las políticas de conocimiento dominantes para tratar de comprender el fondo en el que se inscribe nuestra práctica; las formas de ver el mundo, la escuela y la enseñanza, y cómo están condicionadas por una serie de aspectos discursivos y de acción que vamos incorporando en nuestras vidas, muchas veces sin darnos cuenta de ello. Como docentes, pensamos que si comprendemos, desnaturalizamos y repensamos nuestras nociones y prácticas de inclusión, podríamos favorecer y ampliar diversas posibilidades educativas, ya que dichas nociones pueden afectar nuestra percepción sobre los estudiantes, así como a las estrategias y prácticas tanto formativas como de enseñanza- aprendizaje.

\section{El ciclo indagación-acción-transformación para evitar la exclusión educativa}

Reconocemos que cualquier propuesta para mejorar nuestro accionar pedagógico se enfrenta a múltiples condiciones estructurales y a una compleja dinámica del aula por lo que hemos revisado las características de las políticas educativas neoliberales que han afectado a todos los sistemas educativos en la región latinoamericana. En particular intentamos comprender el papel que dichas políticas han jugado en regular la cultura de nuestra institución, la formación de los alumnos, la práctica docente, la administración escolar y el desarrollo curricular. Por otro lado, hemos observado los aspectos que limitan la formación de colectivos docentes para fomentar procesos de reflexión y construir alternativas conjuntas en relación a la práctica educativa.

Pensamos, apoyados en Skliar (2005), que para poder cuestionar el abordaje a diversidad, como sinónimo de exclusión, marginación y pobreza era necesario descartar la noción que ubica al otro en un lugar de poca jerarquía y analizar los sentidos construidos sobre la categorización de exclusión/ inclusión, por los efectos sobre nuestra visión, división del mundo social y orientación de nuestra actividad.

Sabemos que el proceso de inclusión es un proyecto político fundamental, cuyo tratamiento a la diversidad nos llevaría a considerar los aspectos macro y micro estructurales, sociales, culturales, educativos, políticos, etcétera lo cual rebasa el propósito del artículo. En esta ocasión sólo mencionaremos nuestra inquietud de pensar cómo concretar y transformar nuestras prácticas, visiones y relaciones pedagógicas con los estudiantes y el proceso formativo en general, en el día a día para no quedarnos en el discurso del cambio, que nos permita comprender la complejidad de las narativas que como docentes hacemos de los conflictos y dilemas en nuestra vida cotidiana universitaria (Bolívar, Domingo y Fernández, 2001).

Ahora sabemos que los procesos de exclusión no se dan sólo a nivel de los sujetos, sino se excluyen o marginan prácticas, modos de pensar y maneras de conocer de grupos o sociedades. Además que la experiencia social en todo el mundo es mucho más amplia y variada de lo que la tradición científica o filosófica occidental conoce y considera importante (Santos, 2008). Este autor, destaca el mérito de Foucault de haber demostrado las opacidades y silencios producidos por la ciencia moderna y su búsqueda de "regímenes de verdad" alternativos, por ello nos adherimos a su intención por identificar otras formas de conocimiento que han quedado marginadas, excluidas y desacreditadas por dicha ciencia moderna y reconocer la existencia de diversos tipos de conocimientos y maneras de conocer. 
En el contexto de indagación más amplio en el que se inscribe el presente estudio, ante la crisis de los modos paradigmáticos establecidos de conocer, hemos visto la pertinencia de integrar modos diferentes a la investigación y racionalidad instrumental de la ciencia moderna ha impuesto como única, a través de integrar en los procesos formativos la investigación hermenéutica narrativa, por ejemplo. Por otra parte hemos participado en redes iberoamericanas y colectivos nacionales que intentan ampliar las opciones a la formación de los docentes, por ejemplo como un movimiento horizontal que no se limita a revisar y aplicar los aspectos técnicos ofrecidos por "expertos" sino en reconocer los saberes reconstruidos mediante procesos de indagación-reflexión sobre nuestra práctica y experiencia docente. De igual forma hemos discutido alternativas y opciones retóricas en la difusión del conocimiento, en nuestro caso el saber pedagógico, en foros académicos y publicaciones. Lo hemos hecho participando en discusiones y propuestas sobre modos de ampliar la codificación de la retórica de la investigación "positivista", que según Zeller (2012) encontramos en el Manual de Publicación de APA, que se ha constituido en el manual de estilo para gran parte de las ciencias sociales. Argumentamos contra estas convenciones, que intentan crear la apariencia de objetividad, y que imponen el uso del punto de vista en tercera persona, énfasis en la precisión, evitar el uso de metáforas o usos expresivos del lenguaje y apoyar las afirmaciones en pruebas experimentales y empíricas. En especial porque desafortunadamente aunque muchos investigadores en ciencias humanas han refutado dicha objetividad no se ha rechazado su influencia sobre sus estilos de redacción (Zeller, 2012). Así no sólo diferimos de la investigación tradicional en cómo se construye el conocimiento, sino que asumimos la existencia de perspectivas múltiples en los informes narrativos. Tarea aún pendiente en este sentido ya que no existe una única forma de narrar una historia, de interpretarla, o de escribirla, sino encontramos múltiples formas de acercarnos a la misma, porque el discurso narrativo tiene que ver con relaciones entre particularidades más que entre generalidades abstractas que ha de considerar necesariamente las intenciones y motivaciones humanas (Susinos y Parrilla, 2008).

Congruentes con nuestra postura de considerar la existencia de culturas diferentes, formas expresivas de la diversidad humana que tienen su propia presencia eliminamos cualquier sentido construido de superioridad o de inferioridad. Esto es, consideramos que nada, nadie, grupo o sociedad puede establecerse como modelo a través del cual medir cualquier acontecimiento humano, en nuestro caso el educativo.

En relación con la docencia, lo importante para nosotros no ha sido sólo entenderla sino intentar cambiarla, pero pensamos que este tipo de cambio no surge de la experimentación, observación y la adopción de un punto de vista aparentemente neutral, sino surge desde nuestro propio cambio, a través de una nueva descripción de la práctica (Mc Ewan (2012). Una descripción en la que explicamos las insuficiencias de nuestras visiones y trazamos vías alternas para nuestro quehacer educativo. La reflexión sobre nuestra práctica nos ha servido para expresar lo que hacemos, por qué lo hacemos y para adoptar una postura crítica. Por tal motivo nos referiremos a continuación a las reflexiones expresadas en torno a las relaciones pedagógicas con el estudiantado y en particular con la otredad. 


\title{
2.1. Reflexiones de experiencias docentes sobre la diversidad y propuestas sobre la otredad
}

Iniciamos cuestionando el proceso de naturalización frecuente en nuestras aulas, que consiste en atribuir causas naturales a hechos sociales y que impide problematizar los aspectos sociales que condicionan y orientan nuestro comportamiento. Por ello, a través de la documentación de experiencias pedagógicas y las reflexiones colegiadas con otros docentes, pudimos retomar una vía para reflexionar sobre las apuestas de cambio hacia prácticas educativas inclusivas. Lo hicimos mediante un proceso de desnaturalización y cuestionamiento de algunos de los supuestos y representaciones que orientan muchas de nuestras decisiones y prácticas cotidianas (Skliar, 2005).

Afortunadamente, pudimos acercarnos a las perspectivas de Freire (1997), Sennett (2000) y Bourdieu (2000), entre otros, que plantean una dialéctica entre las condiciones objetivas y simbólicas de producción de la subjetividad al poner en relación los límites o determinaciones objetivas y las esperanzas o expectativas subjetivas. De esta manera, aún reconociendo los determinantes externos, pensamos que la universidad y nosotros mismos podemos actuar sobre los límites objetivos que parecen sentenciar a los estudiantes, contribuyendo a forzar el sentido de los límites subjetivos, es decir, ayudando a los estudiantes a no ajustar mecánicamente sus deseos y horizontes a los límites que les son dados y esperables por su condición social.

Este proceso desnaturalizador ha sido fundamental para comprender cómo en el proceso escolar cotidiano hemos participado en procesos de exclusión, posiblemente sin darnos cuenta, al tratar de reproducir una identidad común y al haber asimilado la idea de una educación universal, homogeneizadora la cual ha dado lugar, entre otras cosas, a ignorar o invisibilizar las diferencias, debido al uso de clasificaciones que desvalorizan a aquellos estudiantes que no se acercan a este modelo "normalizador". Veamos las reflexiones de una colega docente del grupo como parte de su relato con alumnos de preparatoria:

\begin{abstract}
Al no saber cómo proceder, consideré viable compartir la problemática con algunos de mis colegas de mayor experiencia, los cuales externaron comentarios como los siguientes: "Los alumnos flojos y mal educados no pueden estar en el salón, hasta que aprendan a comportarse". "Te recomiendo sacar del aula a los más latosos y con eso el grupo se tranquiliza". "No te preocupes, estamos en una universidad de masas, así que la mayoría va a reprobar". "Su comportamiento refleja su medio, no puedes hacer mucho". "Dedícate con los que si quieren estudiar y ser alguien en la vida. (Mendoza, 2014, p. 21)
\end{abstract}

Reflexiones como la anterior, han podido ayudarnos a hacer visibles las conceptualizaciones y supuestos, compartidos socialmente o que hemos ido formando en nuestra historia docente con nuestros estudiantes, en especial de zonas pobres, para poder resignificarlas y que a su vez ayuden a transformar nuestra práctica. Esto es, recuperar en palabras de Skliar (2005, p.12):
(...) aquellos argumentos que si bien respiran cómodamente en las leyes y en los textos, ellos también suelen hablar en nosotros mismos, nos conminan a argumentar siempre acerca de la imperiosa necesidad de la escuela, nos obligan a ser, nosotros mismos, argumentos vivos de esos argumentos.

De esta manera comprender, como la profesora citada, que algunos problemas de exclusión que se presentan en las aulas se originan en las relaciones entre todos los que participamos, ya que estos problemas se construyen y se resignifican en un proceso 
interactivo, como se observa en el proceso reflexivo de dicha colega continuando con el relato anterior:
(...) Dicha frase provocó que el proceso de reflexión e indagación en el que estuve inmersa, por fin tuviera frutos. Así que preparé una charla con el grupo acerca del concepto que ahora había construido de ellos, como personas inteligentes y privilegiadas porque seguían estudiando y estaban a un paso de ingresar a la universidad. Continúe enfatizándoles que esperaba más de todos, ya que eran los futuros científicos como físicos o matemáticos, que en sus manos estaba por ejemplo, el diseño y creación de una nueva propuesta de vivienda como arquitectos $e$ ingenieros y que por tal motivo les exigiría más. La nueva percepción que ahora tenía hacia el grupo, tuvo impacto positivo porque desperté su inquietud en relación a los objetivos que se pueden llevar a cabo en sus respectivas carreras con relación a la Psicología (...). (Mendoza, 2014, p. 23)

Lo anterior no implica que sólo la escuela o los educadores seamos responsables de esta problemática, sino que reconocemos que en las instituciones educativas podemos colaborar hacia este proceso inclusivo. Por ello, consideramos además cuestionar cómo en las escuelas hemos producido prácticas diferenciales entre ciertos grupos de estudiantes; como sucede a tantos estudiantes, como el que se sinceró con otra de nuestras colegas.

(...) $Y$ volvió a decir, yo no puedo hablar mal de la maestra... En ese momento intuí que había algo que no se atrevía a decir, y le comenté: bueno, la conozco de vista, pero en realidad nunca la he tratado, ni he tenido ningún acercamiento con ella. En ese momento, como que respiró más aliviado, y dijo, - a mí se me hizo una buena maestra, con buenas estrategias, pero como persona,...no sé,... sí... se me hizo muy dura en sus comentarios, a mí me destrozaron. Me dijo que pensara si no estaba perdiendo el tiempo, si no me había equivocado de carrera, si valía la pena que continuara, entre otras cosas, y... continuó: Yo sí me fui para abajo con todo lo que ella me dijo, y opté por abandonar mis estudios. Pensé que efectivamente, no tenía caso, que de verdad, yo no servía, que estaba perdiendo el tiempo, o se los estaba haciendo perder a otros quizá, pero afortunadamente con muchos esfuerzos estoy nuevamente retomando mi carrera, y también pensé no le voy a dar gusto a la maestra, y bueno, aquí estamos. (Nieves, 2014, p. 97)

Este es un botón de muestra, de prácticas excluyentes, que pasan desapercibidas por los docentes. Pero, si nos damos tiempo de tomar distancia de nuestras experiencias y prácticas cotidianas, aprovecharemos la posibilidad de reflexionar sobre ciertos aspectos que obviamos por continuar organizando nuestras prácticas de forma homogénea, negando la complejidad, contradicciones y diversidad de la experiencia escolar cotidiana. Las reflexiones de dicha colega, ante el acontecimiento anteriormente descrito le posicionan ante el hecho educativo de otra forma:

Este breve pasaje me ha hecho pensar muchas cosas, y me asaltaron varias dudas, por ejemplo: ¿Habrá ex alumnos míos por allí que cuenten algo similar de mí? Habré causado un impacto de esa naturaleza en alguien? ¿Cómo me recordarán? $\Upsilon$ mi respuesta es, no lo sé, ya que como dije al principio, la gran mayoría de veces, los comentarios que hacemos son bien intencionados, sin embargo, generalmente no corroboramos que esto haya sido así. Aunque tratamos de suavizarlos, no sabemos el real impacto que causarán y creo que esto tiene que ver por supuesto con la historia de cada quien, con sus circunstancias específicas, con su contexto, que en ocasiones no nos damos el tiempo de indagar, que vemos a todos los alumnos de una manera homogénea que los estandarizamos con una medida que tenemos implícita, cuando en realidad, y lo sabemos, cada uno tenemos diferencias individuales, algo propio, algo que nos distingue como personas, algo y mejor dicho muchas situaciones que nos hacen únicos, y entonces ¿̇cómo resolver todo eso? Lo cierto es que a la hora de estar frente a nuestros alumnos, parece que se nos olvida, como si no tuvieran importancia 
esa historias de vida individuales, esos contextos específicos, y nos avocamos a los criterios establecidos, a lo que nos demanda la institución, a lo que consideramos que debe ser (...). (Nieves, 2014, pp. 97-98)

Con estas interrogantes intentamos desnaturalizar otro supuesto: los problemas en la escuela son de los estudiantes, no del dispositivo escolar, ni de las relaciones escolares, ni de las condiciones pedagógicas (Montesinos, 2005). Con frecuencia los profesores podemos pensar que ciertas condiciones de los alumnos son limitaciones sin solución, y que son determinantes de las posibilidades de aprendizaje de los alumnos. Por lo general, son percibidas como aspectos inherentes al estudiante, que los coloca en una situación de desventaja y que demanda estrategias pedagógicas específicas, como las reflexiones que | nos comparte otra colega:

(...) La experiencia me ha hecho reflexionar sobre la gran variedad de obstáculos
que se pueden presentar a los alumnos en el transcurso de sus estudios debido a la
etapa de la vida en la que se encuentran. Esos obstáculos tienen como consecuencia
discontinuidad de los estudios, reprobación y deserción. Hay quienes tienen
problemas laborales o familiares, por ejemplo padres o hijos que necesitan cuidados
especiales, problemas económicos, problemas de salud, depresión por múltiples causas,
pérdida de trabajo, entorno hostil y en ocasiones dificultades para aprender y hasta
incompatibilidad con compañeros o con profesores; y aún así quieren cumplir con sus
deseos de superación.... (Romo, 2014, p. 11)

Dentro de los múltiples obstáculos que percibimos muchas veces como infranqueables, están las condiciones de pobreza de algunos estudiantes que asisten a las universidades públicas y generalmente son vistas como "riesgo social". En especial, con la certeza de nuestra imposibilidad para modificar dichas condiciones, lo que impide la acción educativa. Por ello, es fundamental pensar en lo contraproducente de dicha visión fatalista, ya que damos por sentado que no podemos educar de la misma manera a los alumnos de sectores marginados, y el problema es que pensemos todo esto, aún antes de que realicemos cualquier intervención pedagógica.

Nuestra propuesta, retomada de Serra y Cancino (2006) es tratar de desnaturalizar la noción de "riesgo" para no utilizarla como un modo diferencial de tratamiento en nuestras relaciones pedagógicas al otorgar destinos sociales y educativos a nuestros estudiantes, ya que si no lo hacemos estaríamos, sin darnos cuenta, utilizando nuevas forma de control desarrolladas en nuestras sociedades, que ya no necesitan ni represión ni el intervencionismo asistencial, sino que se logran mediante la asignación diferencial de un destino social previamente construido. Desde esta lógica, los estudiantes de zonas de pobreza se convierten en sujetos "potencialmente delincuentes", "potencialmente peligrosos", o... "potencialmente desertores del sistema escolar".

Lo relevante con este proceso de reflexión y cambio en nuestra forma de pensar la práctica pedagógica ha sido para: reconocer que las diferencias son una condición inherente a la naturaleza humana; que como especie, cada uno de nosotros tenemos ciertas características que nos hacen diferentes,; que en las aulas de todos los niveles educativos podemos encontrar una enorme diversidad en el alumnado, culturas y prácticas y que estas diferencias debemos reconocerlas y considerarlas en nuestras prácticas cotidianas.

Coincidimos con Florian (2013) es que en lugar de enfocarnos en los criterios diagnósticos referidos para categorizar a los alumnos, atendamos las diferencias educativas, reconocidas en la manera de responder a tareas y actividades académicas. No olvidemos que la valoración negativa de las diferencias, así como los prejuicios pueden 
llevarnos a la exclusión y a la discriminación. Esto es, la diversidad en el ámbito educativo nos remite al hecho de que cada alumno tiene sus propias necesidades educativas y se enfrenta de distinta manera a las experiencias de aprendizaje. Veamos un ejemplo de ello

\begin{abstract}
Un año después, el desarrollo de Alba seguía siendo tan lento que la volví a entrevistar. Le informé que había consumido seis semestres de 10 que tenía como máximo para concluir los estudios y que si ella continuaba acreditando en promedio 2.5 materias por semestre, no podría terminar los estudios a tiempo para tener la oportunidad de graduarse. Por tal motivo la invitaba a reflexionar si era pertinente retirarse, porque estaba invirtiendo tiempo y dinero y finalmente los estudios no serían concluidos. Fue muy difícil para mi plantear la situación porque en ese momento yo pensaba que lo importante era alcanzar la meta, de no hacerlo los estudios no son reconocidos y no cuentan para nada en el desempeño laboral. En ese sentido ¿̨para qué seguir invirtiendo tiempo y dinero? (Romo, 2014, p.10)
\end{abstract}

Casos como los anteriormente narrados, nos han llevado a cuestionar y transformar nuestra práctica y a nosotros mismos. Como hemos mencionado mediante un proceso reflexivo, propio de la edición de los relatos de experiencias pedagógicas, fuimos poco a poco siendo conscientes que este proceso de desnaturalización no es sencillo puesto que implica un cierto distanciamiento y una visión autocrítica de nuestras prácticas cotidianas arraigadas en nuestro sentido común, como señala Skliar (2005), cuya mirada sobre la inclusión nos orienta hacia una pedagogía para la diferencia.

\title{
2.2. Esbozos posibles sobre relaciones pedagógicas para la diferencia u otredad
}

Freire (1997) nos ofreció importantes aportes para pensar posibles relaciones pedagógicas para la diferencia al enfatizar el carácter político y ético de la educación, así como su papel transformador (ver Fernández, 2008). En especial, recuperamos su propuesta de relación dialógica, es decir, el encuentro entre los seres humanos mediatizados por el mundo para leerlo y pronunciarlo; para una educación emancipadora que favorezca la reflexión en sus relaciones con el mundo, cuestionando y problematizando la vida cotidiana de ambos, para favorecer su inclusión crítica en la realidad.

La confianza dirigida y otorgada al otro, que nos propone Freire (1970 y 1997), ha favorecido que reconozcamos y reconocernos en el otro. Según este pensador brasileño, tanto el educador como el educando podemos iniciar el proceso de liberación a través de la praxis, es decir, a través de la reflexión junto con nuestra acción sobre el mundo para su transformación.

Con el pensamiento de Freire (1997) hemos defendido una igualdad que incluye el derecho que tienen todas las personas, grupos y pueblos a vivir sus propias opcione; opuestos a una igualdad homogeneizadora que supone, en la práctica, la imposición de una cultura única a todos los pueblos y personas; contra una diversidad y diferencia que deja en segundo plano la igualdad de derechos de todas las personas y grupos. Nos adherimos al objetivo freiriano, de unidad en la diversidad o igualdad de las diferencias, el cual supone que todo el mundo, sin distinción de clase social, género, cultura o edad, tenga derecho a una educación igualitaria. Desde su óptica la diversidad no es el objetivo sino el camino para llegar al verdadero objetivo que es la igualdad (Aubert, Duque, Fisas y Valls, 2013).

Pero más que el diálogo o el sentido de comunidad, lo que hace que la pedagogía de Freire sea una pedagogía para la diferencia u otredad, es la utilización de la educación 
como medio para que "esos otros" tengan voz, es decir que los estudiantes, pobres, discapacitados, obreros, mujeres, indígenas, campesinos... en suma, los grupos negados, invisibilizados y violentados de nuestra sociedad, tengan la posibilidad de contar su historia y de cambiar su situación (Santo, 2010). Una pedagogía de la diferencia en la que, como propone Skliar (2005), podamos ir al encuentro con el otro, con sus palabras, con su cuerpo, con su mirada, que reconozca la singularidad de los sujetos y sus "alteridades inesperadas" y como advierte Tedesco (1995), se promueva "el vínculo entre los diferentes" mediante el diálogo y el intercambio para permitir "a cada uno elegir y construir sus múltiples identidades." En el ámbito pedagógico, el "encuentro con el otro" abre un territorio de reflexión y toma de decisiones.

El siguiente, es un ejemplo más de reflexión, de reconocimiento y de voz del otro, de experiencias pedagógicas enriquecedoras que aquí les compartimos:

\begin{abstract}
(...)A pesar de que Alba, con todo y su enfermedad de pronóstico negativo, logró acreditar 23 materias de un total de 27 y elaboró su tesis, en el registro escolar su desempeño quedó como una alumna que abandonó los estudios. Es evidente la falta de sensibilidad que los instrumentos de evaluación institucional muestran. Cuando observo las historias académicas de todos esos estudiantes irregulares que tal vez nunca concluyan la especialidad pienso en Alba y en todas esas historias de vida no contadas, que ante las normas de la institución son solamente una estadística negativa para la Universidad, un fracaso escolar ipero pienso que no pueden ser eso! Una persona como Alba que en circunstancias tan extremas trata de aprovechar hasta el último instante para alcanzar su anhelo de aprender como un medio de su expresión de vida, es mucho más que la obtención de un título universitario, es la expresión de ;un ser humano! (Romo, 2014, p. 14)
\end{abstract}

Estas reflexiones, nos ha hecho conscientes en el colectivo de las bondades de la narrativa, tanto como proceso de indagación como de experiencia pedagógica y de construcción de identidad, donde reconocemos al otro y nos reconocemos a nosotros mismos, veamos la voz de una estudiante:

\begin{abstract}
En la presente narrativa, describo mi experiencia pedagógica en el primer comité tutorial de la maestría en pedagogía, debo decir, ya desde un principio que fue una vivencia fuerte, que me dejo más de una lección de vida en lo personal y en lo académico. Luego de un liberador ejercicio de catarsis, a manera de narrativa compartida y comentada en grupo, en el que pude desahogar mi confusión, molestia, y frustración, luego de ello pude librarme de la carga emocional negativa que tal evento me produjo(...) Tal ejercicio renovó positivamente la impresión que tenia del profesorado del posgrado, conciliando, lo que hasta entonces crei discrepancia entre su discurso y sus actitudes, y más aun considerando los discursos de los autores, de los contenidos revisados seminario a seminario, y que fueron cuidadosamente seleccionados por ellos. Lo más importante fue poder cerrar este episodio evidenciando el lugar fundamental que tiene la mediación del lenguaje para saber ampliar enfoques, saber del otro, dando derecho de réplica y negociando nuevos significados de un mismo evento, lo cual aclara, enriquece y fortalece mi experiencia pedagógica de dicho evento y me posiciona desde otra postura menos vulnerable para lo que este por venir en tal contexto (...). (Jazmín, cit. por Olmos, en Prensa, p. 82)
\end{abstract}

Estas reflexiones y esbozos de propuestas son una modesta aportación en vías de construcción de una nueva narrativa como profesores universitarios, donde podamos ir cuestionando lo naturalizado e invisible y que nos permita imaginar escenarios educativos alternativos y de discusión sobre cómo generar en ese proyecto conjunto el mundo y universidad que queremos construir. 


\section{Consideraciones finales}

A través de la serie de reflexiones expuestas, y otras que se quedaron en el tintero o mejor dicho el teclado, hemos deseado expresar las innumerables dudas, así como las pocas certezas, siempre provisionales, sobre nuestro indagar-accionar en relación a cómo atender la diversidad de prácticas, formas de conocer y actuar universitarias. Esta iniciativa nos ha parecido fundamental porque nos ha permitido avanzar en nuestra búsqueda de modos alternativos de mirar y mirarnos, de formar y de formarnos, de investigar, de construir saberes pedagógicos que ofrezcan opciones pedagógicas a la diversidad universitaria.

Hemos considerado importante construir una visión sobre la inclusión educativa, aunque por el momento histórico sólo de los grupos heterogéneos y privilegiados que conforman nuestras universidades, que se desligue de los argumentos formulados por los teóricos de la discapacidad, o de la compensación para atender los intereses y reivindicaciones de todos los individuos o colectivos, que se descentre de los sujetos y se enfoque en el papel de las instituciones para ofrecer a todos los estudiantes múltiples alternativas y opciones pedagógicas; pensada como un movimiento para lograr una educación para todos, con acceso, participación y logro del estudiantado en general, sin olvidar aquellos grupos tradicionalmente excluidos o marginados pero sin clasificarlos y segregarlos. En nuestra opinión, este es un camino difícil sobre el que aún tenemos mucho que recorrer, en especial por la racionalidad instrumental de la ciencia moderna que permea en las tradiciones académicas que han determinado cómo se construye el saber y cómo se parcela el conocimiento que han llevado a visiones parciales, segmentadas y especializadas de lo social.

La reflexión horizontal entre docentes, que se construye con la documentación narrativa de experiencias pedagógicas y su posterior meta-reflexión, puede ayudarnos a constituir un espacio colectivo para reexaminar y replantear por medio de la ruptura o desnaturalización de supuestos, discursos y prácticas que construimos en la cotidianidad del aula. Este proceso de distanciamiento y reflexión puede apoyarnos para vislumbrar las posibilidades que hemos pasado por alto para transformar nuestras prácticas y supuestos educativos, recordando que todos somos diferentes y aprovechar dicha riqueza y diversidad en los procesos formativos de todos los implicados.

Este diálogo y enseñanza recíproca entre colegas, a través de narrativas pedagógicas o de vida, puede favorecer la revisión, re-definición y compartir prácticas educativas que se orienten al desarrollo de una cultura incluyente, que no minimice los contextos en que se desarrolla el estudiante, ni las relaciones sociales y pedagógicas que puedan limitar o apoyar una educación para todos, a través de una pedagogía para la diferencia u otredad que reconoce y da voz a todos, en especial a los larga e históricamente excluidos.

Finalmente, consideramos que los relatos de experiencia pedagógica y la reflexión sistemática pueden servirnos como instrumento de resistencia y transformación, ya que favorecen que imaginemos actuaciones docentes y mundos posibles liberados de las representaciones de la universidad tradicional, al que el discurso racional nos ha encadenado y que han impedido abrir un camino de transformación del orden establecido. Pensamos que mediante esas pequeñas pero ricas narrativas docentes podremos abrir la posibilidad de imaginar y soñar el cambio anhelado en nuestras aulas universitarias. 


\section{Referencias}

Ainscow, M. (2012). Haciendo que las escuelas sean más inclusivas: lecciones a partir del análisis de la investigación internacional. Revista de Educación Inclusiva, 5(1), 39-49.

Aubert, A., Duque, E., Fisas, M. y Valls, R. (2013). Dialogar y transformar. Pedagogía crítica del siglo XXI. Barcelona: Editorial Grao.

Blanco, R. (2006). La equidad y la inclusión social. Uno de los desafíos de la educación y la escuela de hoy. REICE. Revista Iberoamericana sobre Calidad, Eficacia y Cambio en Educación, 4(3), 115 .

Bolívar, A., Domingo, J. y Fernández, M. (2001). La investigación biográfico-narrativa en educación. Enfoque y metodología. Madrid: La muralla.

Bourdieu, P. (2000). Poder, derecho y clases sociales. Bilbao: Desclée de Brouwer.

Castel, R. (2004). Las trampas de la exclusión. Trabajo y utilidad social. Buenos Aires: Topia Editorial.

Duhalde, M. (2008). Pedagogía crítica y formación docente. En M. Godotti., M. Gómez., M. Jason y A. Fernandes (Comps.), Paulo Freire. Contribuciones para la pedagogía (pp. 201-213). Buenos Aires: CLACSO.

Fernández, M. (2008). Hacia una pedagogía de las diferencias desde los aportes de la propuesta de Paulo Freire. En M. Godotti., M. Gómez., M. Jason y A. Fernandes (Comps.), Paulo Freire. Contribuciones para la pedagogía (pp. 341-347). Buenos Aires: CLACSO.

Florian, L. (2013). La educación especial en la era de la inclusión: ¿El fin de la educación especial o un nuevo comienzo? Revista Latinoamericana de Inclusión Educativa, 7(2), 27-36.

Freire, P. (1970). Pedagogía del oprimido. Buenos Aires: Siglo XXI.

Freire, P. (1997). Pedagogía de la autonomía. Buenos Aires: Siglo XXI.

Kaplan, C. y García, S. (2006). La inclusión como posibilidad. Buenos Aires: Ministerio de Educación, Ciencia y Tecnología de la Nación.

Kincheloe, J. (2001). Hacia una revisión crítica del pensamiento docente. Barcelona: Octaedro.

McEwan, H. (2012). Las narrativas en el estudio de la docencia. En H. McEwan y K. Egan (Comps.), La narrativa en la enseñaza, el aprendizaje y la investigación (pp. 236-259). Buenos Aires: Amorrortu editores.

Mendoza, A. (2014). Un grupo rebelde con causa que fortaleció mi práctica docente. En A. Olmos y L. Arias (Coords.), Relatos de experiencias pedagógicas universitarias (pp. 13-21). Ciudad de México: UNAM.

Mezirow, J (1998). Concepto y acción en la educación de adultos. En J. Sáez Carreras y A. Escarbajal de Haro (Coord.), La educación de personas adultas. En defensa de la reflexividad cívica (pp. 25-36). Salamanca: Amaru.

Montesinos, M. (2005). Notas sobre la producción del fracaso escolar masivo. En OEA. (Ed.), Experiencias pedagógicas: voces y miradas. estrategias y materiales pedagógicos para la retención escolar (pp. 192-205). Buenos Aires: Ministerio de Educación, Ciencia y Tecnología de la Nación.

Nieves, R. (2014). El impacto de los comentarios del docente a sus alumnos ¿Los estimulamos, o sencillamente los bloqueamos. En A. Olmos y L. Arias (Coords.), Relatos de Experiencias Pedagógicas Universitarias (pp. 78-84). Ciudad de México: UNAM. 
Reflexiones Docentes sobre Inclusión Educativa. Relatos de Experiencia Pedagógica...

Olmos, A. (En Prensa). La narrativa pedagógica y el proceso de formación. Fundamentos del dispositivo metodológico. En A. Olmos y L. Figueroa (Eds.). Reflexiones y transformaciones docentes (pp. 13-46). Ciudad de México: UNAM.

Romo, R. (2014). Aprender es una expresión de la vida. Lo que aprendí de Alba. En A. Olmos y L. Arias (Coords.), Relatos de Experiencias Pedagógicas Universitarias (pp. 5-12). Ciudad de México: UNAM.

Rosenthal, R. (1994). Interpersonal expectancy effects: A 30- year perspective. American Psychologhy Society, 3(6), 176- 179. doi:10.1037/e511112010-003

Saavedra, M; González, V y López, M. (2005). Los problemas de la formación: marco epistémico. En M. Gómez y H. Zemelman (Coords.), Discurso pedagógico. Horizonte epistémico de la formación docente (pp. 10- 32). Ciudad de México: Editorial Pax.

Santo, M. (2010). Pedagogía, historia y alteridad. Teoría de la Educación, 22(2), 63-84.

Santos, B. (2006). Conocer desde el sur. Para una cultura política emancipatoria. La Paz: CLACSO.

Santos, B. (2008). Pensando el Estado y la sociedad: desafíos actuales. La Paz: Muela del Diablo Editores.

Sennett, R. (2000). La corrosión del carácter. Las consecuencias personales del trabajo en el nuevo capitalismo. Barcelona: Anagrama.

Serra, S. y Canciano, E. (2006). Las condiciones de enseñanza en contextos críticos. Buenos Aires: Ministerio de Educación, Ciencia y Tecnología de la Nación.

Skliar, C. (2005). Poner en tela de juicio la normalidad, no la anormalidad. Políticas y falta de políticas en relación con las diferencias en educación. Revista Educación y Pedagogía, 17(41), 11-22.

Suárez, D. (2006). Documentación narrativa de Experiencias pedagógicas. Una manera de indagar el mundo y las experiencias escolares.Entre Maestr@s,5(16),37-51.

Suárez, D. y Ochoa, L. (2005). La documentación de experiencias pedagógicas. Una estrategia para la formación de docentes. Buenos Aires: MECYT/OEA.

Susinos, T. y Parrilla, Á. (2008). Dar la voz en la investigación inclusiva. Debates sobre inclusión y exclusión desde un enfoque biográfico-narrativo. REICE. Revista Iberoamericana sobre Calidad, Eficacia y Cambio en Educación, 6(2), 157-171.

Tedesco, I. (1995). El nuevo pacto educativo: Educación, competitividad y ciudadanía. Madrid: Anaya.

Zeller, N. (2012). La racionalidad narrativa en la investigación educativa. En H. McEwan y K. Egan (Comps.), La narrativa en la enseñanza, el aprendizaje y la investigación (pp. 295-334). Buenos Aires: Amorrortu editores.

\section{Breve CV de las autoras}

\section{Andrea Olmos Roa}

Doctora en Comunicación, Cultura y Educación, Fac. Estudios Sociales, Universidad de Salamanca, España. Maestra y Lic. Psicología, Universidad Nacional Autónoma México. Profesora del Posgrado (Psicología y Pedagogía). Libros: Olmos, A. y Martínez, M. (2013). Identidad y Narrativa: Recuperando voces de adolescentes y docentes. México: UNAM/FESZ; Tirado y otros (2010). Psicología educativa para afrontar los desafíos del siglo XXI. México: McGraw-Hill; Olmos, A. y Carrillo, A. (2009). Juego y 
alfabetización. Bases para un diseño educativo cultural vygotskiano. México: UNAM/Porrúa; Acle, G. y Olmos A. (1998). Problemas de Aprendizaje: Enfoques Teóricos. México: UNAM. Responsable Académico Proyecto Investigación (PE204114) Narrativa y Reflexión pedagógica, su impacto en la enseñanza e Innovación en aulas universitarias. DGAPA, UNAM (2014-2016). Email: andreaolmos@gmail.com

\section{María Rebeca Romo Pinales}

Maestra en Odontología, U. Nacional Autónoma de México, Cirujano Dentista, UNAM. Diplomada en Educación a Distancia, CUAED, UNAM. Profesora del Posgrado en la Facultad de Estudios Superiores, Zaragoza, UNAM. Coordinadora de la Especialización en Estomatología en Atención Primaria. División de Investigación y Estudios de Posgrado, UNAM. Email: romorp@servidor.unam.mx

\section{Laura María del Carmen Arias Vera}

Licenciatura en Odontología, Facultad de Odontología, Universidad Nacional Autónoma de México (UNAM). Maestría en Educación Superior, Facultad de Filosofía y Letras, UNAM; Diplomado en Investigación Clínica y Epidemiológica, FES Zaragoza, UNAM; Coordinadora del tercer año de la carrera de Cirujano Dentista de la FES Zaragoza ( 2013); Secretaria Técnica de la Carrera de Cirujano Dentista de la FES Zaragoza (2013). Email: ariaslaura73@yahoo.com.mx 